\title{
Primary Care Physician Perspectives on Recommending E-cigarettes to Smokers: a Best-Worst Discrete Choice Experiment
}

\author{
Ramzi G. Salloum, $P h D^{7}$ (D) , Jennifer H. LeLaurin, MPH ${ }^{7}$, Ji-Hyun Lee, DrPH²,3, \\ Jennifer Elston Lafata, $P h D^{4}$, Maribeth Williams, MD ${ }^{5}$, Yu Wang, $M S^{2}$, \\ James M. Smith, MD', Stephanie A. S. Staras, $P h D^{7}$, Scott M. Strayer, MD, MPH ${ }^{7}$, and \\ James F. Thrasher, $P h D^{8}$
}

\begin{abstract}
'Department of Health Outcomes and Biomedical Informatics, College of Medicine, University of Florida 2004 Mowry Road, Gainesville, FL, USA; ${ }^{2}$ Division of Quantitative Sciences, University of Florida Health Cancer Center Gainesville, USA; ${ }^{3}$ Department of Biostatistics, College of Public Health and Health Professions \& College of Medicine, University of Florida Gainesville, USA; ${ }^{4}$ Division of Pharmaceutical Outcomes and Policy, UNC Eshelman School of Pharmacy and UNC Lineberger Comprehensive Cancer Center, University of North Carolina at Chapel Hill Chapel Hill, USA; ${ }^{5}$ Department of Community Health and Family Medicine, College of Medicine, University of Florida Gainesville, USA; ${ }^{6}$ Division of General Internal Medicine, Department of Medicine, College of Medicine, University of Florida Gainesville, USA; ${ }^{7}$ Department of Family Medicine and Population Health, School of Medicine, Virginia Commonwealth University Richmond, USA; ${ }^{8}$ Department of Health Promotion, Education, and Behavior, Arnold School of Public Health, University of South Carolina Columbia, USA.
\end{abstract}

BACKGROUND: Recent clinical trials suggest that ecigarettes may be more effective for smoking cessation than traditional cessation aids, yet primary care physician (PCP) practices regarding e-cigarette recommendations for smokers have not been studied in-depth.

OBJECTIVE: To identify factors influencing PCP recommendation of e-cigarettes for smoking cessation.

DESIGN: Discrete choice experiment and survey.

PARTICIPANTS: Florida PCPs.

MEASURES:The survey included a discrete choice experiment in which PCPs indicated whether they would recommend e-cigarettes for each of 8 hypothetical patient profiles with the following contrasting characteristics: ecigarette use, interest in approved cessation methods, smoking intensity, prior experience with approved cessation medications, quit intention, age, and comorbidity. Responses were summarized using descriptive statistics and standardized scores (SS).

KEY RESULTS: The sample $(n=216)$ was predominately male (76\%), white (66\%), and non-Hispanic (78\%), and most respondents had held their medical degree for $20+$ years (77\%). The response rate was $28.7 \%$. Most PCPs thought e-cigarettes were at least somewhat effective for smoking cessation (66\%) and lowering disease risk (65\%); $31 \%$ perceived e-cigarettes to be equally/more effective than traditional cessation aids. PCPs were split regarding whether e-cigarettes were less (50\%) or equally harmful (38\%) as cigarettes. Yet, few were very confident in their ability to counsel patients on e-cigarettes risks $(27 \%)$ or benefits (15\%). PCPs recommended e-cigarettes in $27 \%$ of patient profiles they evaluated. The most important factors influencing the decision to recommend or not

Prior Presentations A version of this paper will be presented at the Annual Conference of the Society for Research on Nicotine and TobaccoEurope on September 18, 2020.

Supplementary Information The online version contains supplementa ry material available at https://doi.org/10.1007/s11606-021-06615-w.

Published online February 1, 2021 recommend e-cigarette were patients' prior use of nicotine replacement therapy with $(\mathrm{SS}=0.22,95 \% \mathrm{CI}=0.17-0.27)$ and without use of other medications for cessation (SS = $0.18,95 \% \mathrm{CI}=0.13-0.23$ ), and being middle age (50 years old) with chronic obstructive pulmonary disease (SS = $0.16,95 \% \mathrm{CI}=0.10-0.23$ ).

CONCLUSIONS: Considering the increased patient use of e-cigarettes and increasing use for cessation, this study highlights the need for guidelines and education to aid PCPs' counseling of patients about e-cigarette use.

KEY WORDS: e-cigarettes; primary care; smoking cessation.

J Gen Intern Med 36(11):3353-60

DOI: $10.1007 / \mathrm{s} 11606-021-06615-\mathrm{w}$

(c) Society of General Internal Medicine 2021

\section{INTRODUCTION}

The popularity of e-cigarettes has grown in recent years, reaching over eight million US adult current users in 2018. ${ }^{1}$ When smokers try to quit, they are more likely to use ecigarettes than nicotine replacement therapy (NRT) and prescription cessation medications, with $35 \%$ of smokers substituting some e-cigarettes for cigarettes and $25 \%$ switching to e-cigarettes. ${ }^{2}$ Reported reasons for using ecigarettes include using them to quit or reduce consumption of cigarettes and the belief that they are a healthier alternative to cigarettes. ${ }^{3-6}$ There is a growing consensus that e-cigarettes are a significantly less harmful alternative to cigarettes; ${ }^{7-9}$ however, e-cigarettes are not without risks, as they contain toxins and their long-term health effects are yet to be determined. $^{8}$

The effectiveness of e-cigarettes for smoking cessation remains inconclusive, ${ }^{10-12}$ with a recent Cochrane review citing moderate evidence for relative effectiveness over 
NRT. ${ }^{13}$ In a recent clinical trial of smokers who were ready to quit, 1-year sustained abstinence rates were almost twice as high for smokers who were recommended and provided ecigarettes compared to NRT (18.0\% vs. $9.9 \%) .{ }^{14}$ However, the rate of continuing e-cigarette use at 1-year was fairly high, suggesting the potential for ongoing long-term use. Ecigarettes have become the most commonly used cessation strategy in the USA ${ }^{15}$ and have gained popularity among nonUS smokers, as well. Health authorities in the UK currently advocate that physicians promote e-cigarettes among all patients who smoke, whether for harm reduction or smoking cessation. ${ }^{16,17}$ Given the widespread use of e-cigarettes for cessation, ${ }^{2}$ uncertainties around their efficacy for cessation, and their potential health risks, patients should consider associated risks and benefits to make informed decisions regarding their use.

Many smokers are supportive of their primary care physician (PCP) discussing e-cigarettes with them. ${ }^{18}$ In one study, over three-fourths of PCPs reported that their patients who smoke ask them about e-cigarettes at least some of the time, ${ }^{19}$ and $8-27 \%$ of smokers reported discussing e-cigarettes with their healthcare providers. ${ }^{20-22}$ However, less than $10 \%$ of current e-cigarette users reported discussing the benefits and harms of e-cigarettes with their providers. ${ }^{23}$ Most smokers visit their PCP annually ${ }^{24,25}$ and patients view PCPs as their most trusted source of information on the health effects of ecigarettes. ${ }^{26}$ Thus, clinical encounters present an opportunity for PCPs to inform their patients about the relative risks and benefits of e-cigarette use.

Clinical recommendations call for PCPs to screen for tobacco use and discuss cessation with their patients, but the US Preventive Services Task Force has concluded there was insufficient evidence to support a recommendation on e-cigarettes. ${ }^{27}$ While patients are increasingly asking their PCPs about e-cigarettes, many providers report low self-efficacy to counsel patients on these products. ${ }^{19,28,29}$ In one survey, less than half of PCPs reported feeling confident about their knowledge and ability to answer questions about ecigarettes. $^{19}$

Given the limited availability of evidence and guidelines regarding e-cigarettes for smoking cessation, it is not surprising that there is significant variation in e-cigarette recommendation practices among PCPs. ${ }^{21,22,30-32}$ Among US smokers who discuss e-cigarettes with their providers, $40-66 \%$ report receiving a recommendation from their provider to use ecigarettes as a smoking cessation aid. ${ }^{20-22}$ Notably, in one survey of smokers who discussed e-cigarettes with their providers, $25 \%$ reported their providers had no opinion, while $40 \%$ received a recommendation and $33 \%$ reported that their providers advised against using e-cigarettes. ${ }^{22}$

To date, few studies have examined the factors affecting physician recommendations for e-cigarettes. Cross-sectional studies of smokers have found that discussion of e-cigarettes with providers is higher among smokers who use e-cigarettes or are interested in quitting, ${ }^{21-23,33}$ although the directionality of the association is unclear. Younger age, being male, and higher educational attainment have also been associated with receiving physician recommendations to use e-cigarettes, but these associations have not been found in other studies. ${ }^{21,22}$ Qualitative research with clinicians indicates that patient comorbidities and level of addiction influence their recommendations, ${ }^{34}$ with a greater openness to recommending ecigarettes to smokers who also use e-cigarettes and smokers who are uninterested in quitting, for whom a harm reduction approach may be appropriate. ${ }^{35}$

In the present study, we sought to extend the existing knowledge by modeling physician decision-making regarding counseling patients to use e-cigarettes for smoking cessation. We conducted a discrete choice experiment (DCE) with PCPs to identify patient factors influencing recommendation of ecigarettes. Guided by the Consolidated Framework for Implementation Research (CFIR), ${ }^{36}$ we additionally sought to assess PCP practices, knowledge, and attitudes regarding ecigarettes to inform future clinic-based interventions that address the role of e-cigarettes in smoking cessation.

\section{METHODS}

We conducted a statewide survey of PCPs who treat adult patients, using a DCE to better understand their decisionmaking regarding recommendations to use e-cigarettes for their patients who smoke. The DCE is an established economics methodology based on random utility theory that is increasingly being used in health research ${ }^{37}$ broadly and in ecigarettes research ${ }^{38-40}$ specifically to elicit stakeholder preferences. A recent systematic review concluded that DCEs can produce reasonable predictions of health-related behaviors. ${ }^{41}$ One variant of the DCE, Best-Worst Scaling (BWS), offers advantages to traditional rating or ranking techniques as a lowburden method for quantitatively prioritizing a relatively large number of observed factors. ${ }^{42}$ Rather than only identifying the best alternative, respondents in BWS choose the most preferred item (best) and the least preferred (worst) which provides ratio scales of importance, overcoming concerns that respondents do not use the ratings the same way across responses. This method also overcomes the issue of many items having similar importance weights. We applied the "profile case" BWS in this study to evaluate patient profiles, whereby the same attributes of the patient appear in each scenario, while their values change.

\section{Study Sample and Procedures}

We obtained a specialty-stratified random sample of 801 PCPs in Florida (50\% general internal medicine and 50\% family medicine) from the American Medical Association (AMA) Masterfile: a database maintained by the AMA for the purpose of documenting education and professional practice information for nearly all MDs and DOs in the USA. The AMA Masterfile is the most commonly used sampling frame of 
physicians for health surveys although physicians can opt-out of inclusion. ${ }^{43}$ Individuals were eligible if they were a PCP in Florida seeing adult patients.

To maximize the response rate, we followed the Dillman tailored design method. ${ }^{44}$ PCPs were first mailed a postcard alerting them of and priming them for the study. Approximately a week later, PCPs received the survey via courier with an upfront $\$ 10$ cash incentive and waiver of documentation of informed consent as return of the survey was considered consent. Non-responders received a reminder postcard followed by a second survey mailing. A total of 801 surveys were mailed in two waves - 401 in July 2019 and 400 in December 2019. Of note, the survey administration coincided with the Ecigarette or Vaping Product Use-Associated Lung Injury (EVALI) outbreak when uncertainty about e-cigarettes was high. The study was approved by the University of Florida Institutional Review Board.

\section{Survey Measures}

The survey included the DCE followed by 44 questions assessing PCPs' current e-cigarette recommendation practices, attitudes toward e-cigarettes, clinic characteristics, and PCP characteristics. Survey questions were guided by the $\mathrm{CFIR}^{36}$ and previous surveys on e-cigarettes conducted with physicians. $^{30,31,36}$ The survey, including the DCE, was developed based on interviews with PCPs,${ }^{35}$ with feedback on facevalidity and cognitive testing provided by an interdisciplinary team of tobacco control researchers, physicians, and research assistants.

\section{Experimental Design}

We presented respondents with a series of systematically manipulated profiles of patient characteristics (example in Fig. 1). Each profile consisted of 6 attributes: combinations of age with comorbidities (aged 25 years with no comorbidities, aged 50 years with no comorbidities, aged 75 years with no comorbidities, aged 50 years with previous myocardial infarction $[\mathrm{MI}]$, aged 75 years with previous MI, aged 50 years with COPD, aged 75 years with COPD, aged 75 years with lung cancer); smoking intensity (heavy smoker, light smoker); intention to quit smoking (intends to quit within 30 days, intends to quit within 6 months, intends to quit after 6 months, no intention to quit); experience with cessation methods (tried prescription cessation medications but failed, tried NRT but failed, tried NRT and prescription medications but failed, no experience with approved cessation medications); intention to use approved cessation methods (interested in approved cessation methods, not interested in approved cessation methods); and experience with e-cigarettes (current e-cigarette user, interested in trying e-cigarettes). The DCE used a balanced-incomplete block factorial design with 8 choice sets. Four versions of the DCE were randomly assigned to PCPs. For each choice set, PCPs chose which characteristics were the most and least important in recommending e-cigarettes to the patient.

\section{Statistical Analysis}

Survey responses were summarized with frequencies and percentages, with the exception of age (mean and standard deviation [SD]). We conducted a sensitivity analysis to compare responses received before and after the EVALI outbreak but did not detect differences in relevant measures (results not shown). For the DCE, we observed the total number of times each response item was chosen as most likely and least likely to influence the PCP's recommendation across all choice sets. We obtained a raw score for each response item by calculating the difference between the most and least counts. Since the DCE attributes contained differing numbers of levels, we calculated standardized scores (SS) by dividing the raw score by the maximum possible score ${ }^{45}$ (i.e., number of times the item appeared multiplied with the total number of respondents), in order to allow for comparison of the relative importance of attributes; 95\% confidence intervals (CI) were generated by the bootstrapping method. A stratified analysis was conducted with choice sets that received "yes" and "no" recommendations for e-cigarettes. The analysis was conducted with SAS 9.4 software.

\section{RESULTS}

The response rate for this study was $28.7 \%$, calculated using the American Association for Public Opinion Research guidelines, ${ }^{46}$ and the proportional allocation method to estimate the eligibility rate for unreturned surveys. ${ }^{47}$ We received 216 completed surveys, $84(10.5 \%)$ were undeliverable, 429 (53.6\%) were not returned, 55 (6.9\%) PCPs refused to participate, and 17 (2.1\%) recipients were ineligible. Respondent demographics and clinic characteristics are presented in Table 1 . The sample was predominately male $(75.5 \%)$, white (66.2\%), and non-Hispanic (77.8\%), and most respondents had held their medical degree for over 20 years $(76.9 \%)$. A total of $9(4.2 \%)$ respondents were current tobacco users and 8 (3.7\%) had ever used e-cigarettes.

\section{E-cigarette Knowledge, and Attitudes, and Practices}

Table 2 presents findings on PCP knowledge and attitudes toward e-cigarettes; because response options varied among questions, results are presented on a scale, ranging from least (1) to most (5) favorable views/higher confidence. Generally, attitudes toward e-cigarettes were unfavorable regarding effectiveness for smoking cessation and harm reduction. About half of PCPs were at least somewhat confident in their ability to counsel patients on the benefits (45.4\%) and harms (53.7\%) of e-cigarettes while fewer were not confident at all about their ability to counsel on the benefits (34.7\%) and harms (16.2\%).

About half of PCPs (56.5\%) indicated they were unaware of USPSTF guidelines related to e-cigarettes. Among the 45 (20.8\%) PCPs who indicated their institution/practice had a 


\begin{tabular}{|c|c|c|}
\hline $\begin{array}{l}\text { 1. An adult patient who smokes comes in for a routine visit and asks for your opinion on e-cigarettes. } \\
\text { Check ONE box by the characteristic you consider most important in your decision to recommend } \\
\text { e-cigarettes and the ONE box by the characteristic you consider the least important in your decision to } \\
\text { recommend e-cigarettes. }\end{array}$ & $\begin{array}{c}\text { LEAST important for } \\
\text { e-cigarette } \\
\text { recommendation }\end{array}$ \\
\hline $\begin{array}{c}\text { MOST important for } \\
\text { e-cigarette } \\
\text { recommendation }\end{array}$ & 25 years old with no comorbidities \\
\hline$\square$ & No intention to quit & $\square$ \\
\hline$\square$ & No experience with approved cessation methods \\
\hline$\square$ & Light smoker (5 cigarettes/day) \\
\hline$\square$ & Not interested in approved cessation methods \\
\hline$\square$ & (i.e., nicotine replacement or prescription medications) & $\square$ \\
\hline$\square$ & Current e-cigarette user \\
\hline
\end{tabular}

Figure 1 Example choice set from the discrete choice experiment.

policy on e-cigarette recommendations, $36(80.0 \%)$ indicated the policy discouraged recommendation of e-cigarettes. PCPs' most common source of information on e-cigarettes was professional guidelines or scientific literature $(61.6 \%)$, followed by media $(29.6 \%)$ and patients $(19.4 \%)$. Regarding harm reduction, $44.9 \%$ of PCPs believed that e-cigarettes reduced secondhand smoke exposure and $21.3 \%$ believed they reduce patients' cancer risk. Most PCPs $(67.1 \%)$ believed that ecigarette use sustains nicotine dependence, $43.1 \%$ believed e-cigarettes make patients less likely to use approved cessation methods, and 38.0\% believed e-cigarettes create dual users.

About half of PCPs reported they often or always ask patients about e-cigarette use (Table 3). Less than $10 \%$ of PCPs indicated they recommend e-cigarettes for cessation often or always, while almost half stated they never recommended e-cigarettes for cessation. Most PCPs stated they encourage patients to try other cessation methods before ecigarettes often or always.

\section{Factors Influencing E-cigarette Recommendation}

PCPs endorsed recommending e-cigarettes in one-quarter $(26.9 \%)$ of choice sets, did not recommend them in one-half of choice sets $(51.6 \%)$, and did not provide a response for $21.5 \%$ of choice sets. One-third (33.8\%) of PCPs uniformly indicated they would not recommend e-cigarettes across all 8 patient profiles and $15(6.9 \%)$ stated they would recommend e-cigarettes across all profiles (not shown).

Standardized scores of attribute values from all PCPs, regardless of e-cigarette recommendation, are presented in Figure 2. A patient's past unsuccessful quit attempt using Food and Drug Administration (FDA)-approved cessation medications (i.e., prescriptions and/or NRT) were represented in 3 of the 4 highest-scoring (i.e., most influential) attributes. When PCPs indicated they would recommend e-cigarettes based on the patient profile (not shown), the highest-scoring attribute
Table 1 Provider and Clinic Characteristics $(N=216)$

\begin{tabular}{|c|c|}
\hline Characteristic & $N(\%)$ \\
\hline \multicolumn{2}{|l|}{ Age } \\
\hline Mean (SD) & $58.6(9.1)$ \\
\hline \multicolumn{2}{|l|}{ Gender } \\
\hline Male & $163(75.5)$ \\
\hline Female & $46(21.3)$ \\
\hline Missing & $7(3.2)$ \\
\hline \multicolumn{2}{|l|}{ Race } \\
\hline Asian & $32(14.8 \%)$ \\
\hline Black/African-American & $8(3.7 \%)$ \\
\hline White & $143(66.2 \%)$ \\
\hline Other & $24(11.1 \%)$ \\
\hline Missing & $9(4.2 \%)$ \\
\hline \multicolumn{2}{|l|}{ Ethnicity } \\
\hline Hispanic & $39(18.1 \%)$ \\
\hline Non-Hispanic & $168(77.8 \%)$ \\
\hline Missing & $9(4.2 \%)$ \\
\hline \multicolumn{2}{|l|}{ Year since medical degree } \\
\hline $1-20$ years & $43(19.9 \%)$ \\
\hline$>20$ years & $166(76.9 \%)$ \\
\hline Missing & $7(3.2 \%)$ \\
\hline \multicolumn{2}{|l|}{ Specialty } \\
\hline Family medicine & $98(45.4 \%)$ \\
\hline Internal medicine & $103(47.7 \%)$ \\
\hline Missing & $15(6.9 \%)$ \\
\hline \multicolumn{2}{|l|}{ Percent of time devoted to patient care } \\
\hline $0-49 \%$ & $11(5.1 \%)$ \\
\hline $50-100 \%$ & $196(90.8 \%)$ \\
\hline Missing & $9(4.2 \%)$ \\
\hline \multicolumn{2}{|l|}{ Current tobacco use } \\
\hline Every day/some days & $9(4.2 \%)$ \\
\hline Not at all & $199(92.1 \%)$ \\
\hline Missing & $8(3.7 \%)$ \\
\hline \multicolumn{2}{|l|}{ Ever used e-cigarettes } \\
\hline Yes & $8(3.7 \%)$ \\
\hline No & $202(93.5 \%)$ \\
\hline Missing & $6(2.8 \%)$ \\
\hline \multicolumn{2}{|l|}{ Providers in practice } \\
\hline Median (min, $\max )$ & $3(0,720)$ \\
\hline Mean (SD) & $12.6(58.9)$ \\
\hline \multicolumn{2}{|l|}{ Primary practice } \\
\hline Academic & $3(1.4 \%)$ \\
\hline Hospital-based & $22(10.2 \%)$ \\
\hline Stand-alone (multiple physicians) & $75(34.7 \%)$ \\
\hline Stand-alone (solo practice) & $101(46.8 \%)$ \\
\hline Missing & $10(4.6 \%)$ \\
\hline Other & $5(2.3 \%)$ \\
\hline
\end{tabular}


Table 2 Provider Knowledge and Attitudes on e-Cigarettes $(\mathrm{N}=\mathbf{2 1 6})$

\begin{tabular}{|c|c|c|c|c|c|}
\hline Response level & Least (1) & (2) & (3) & (4) & Most (5) \\
\hline Favorable view of e-cigarettes as a smoking cessation aid ${ }^{a}$ & $63(29.2 \%)$ & $54(25.0 \%)$ & $53(24.5 \%)$ & $31(14.4 \%)$ & $6(2.8 \%)$ \\
\hline Effectiveness as smoking cessation aid ${ }^{\mathrm{b}}$ & $63(29.2 \%)$ & $99(45.8 \%)$ & $31(14.4 \%)$ & $9(4.2 \%)$ & $4(1.9 \%)$ \\
\hline Effectiveness at lowering the risk of tobacco-related disease ${ }^{b}$ & $76(35.2 \%)$ & $84(38.9 \%)$ & $27(12.5 \%)$ & $14(6.5 \%)$ & $5(2.3 \%)$ \\
\hline Harm compared to cigarettes ${ }^{\mathrm{c}}$ & $23(10.6 \%)$ & $85(39.4 \%)$ & $81(37.5 \%)$ & $9(4.2 \%)$ & $8(3.7 \%)$ \\
\hline Effectiveness vs. traditional cessation aids ${ }^{\mathrm{d}}$ & $72(33.3 \%)$ & $68(31.5 \%)$ & $31(14.4 \%)$ & $33(15.3 \%)$ & $4(1.9 \%)$ \\
\hline Patient acceptability vs. traditional cessation aids ${ }^{\mathrm{e}}$ & $43(19.9 \%)$ & $40(18.5 \%)$ & $43(19.9 \%)$ & $64(29.6 \%)$ & $18(8.3 \%)$ \\
\hline $\begin{array}{l}\text { Importance of offering patients alternatives to traditional cessation } \\
\text { treatment }\end{array}$ & $10(4.6 \%)$ & $42(19.4 \%)$ & $31(14.4 \%)$ & $49(22.7 \%)$ & $72(33.3 \%)$ \\
\hline Importance of counseling smokers on e-cigarettes ${ }^{\mathrm{f}}$ & $34(15.7 \%)$ & $49(22.7 \%)$ & $36(16.7 \%)$ & $43(19.9 \%)$ & $42(19.4 \%)$ \\
\hline Confidence to counsel patients on risks of e-cigarettes ${ }^{g}$ & $35(16.2 \%)$ & - & $116(53.7 \%)$ & - & $58(26.9 \%)$ \\
\hline Confidence to counsel patients on benefits of e-cigarettes ${ }^{g}$ & $75(34.7 \%)$ & - & $98(45.4 \%)$ & - & $32(14.8 \%)$ \\
\hline
\end{tabular}

Response options:

(1) very unfavorable to (5) very favorable

${ }^{b}$ (1) not effective to (5) very effective

c(1) much less harmful to (5) much more harmful

d(1) much less effective to (5) much more effective

e(1) much less acceptable to (5) much more acceptable

$f_{(1)}$ not important at all to (5) very important

${ }^{g}(1)$ not confident to (5) very confident

values were as follows: 50 years old with COPD (SS; 95\% CI: $0.27 ; 0.17-0.36$ ), 75 years old with previous MI (SS; 95\% CI: $0.22 ; 0.14-0.32$ ), and those tried to quit using both NRT and prescription medications but failed (SS; 95\% CI: 0.22 ; 0.15 0.29). When PCPs indicated they would not recommend ecigarettes based on the patient profile, the highest-scoring attribute values were as follows: tried to quit using both NRT and prescription medications but failed (SS; 95\% CI: $0.11 ; 0.07-0.16$ ), tried to quit with prescription medications but failed (SS; 95\% CI: $0.09 ; 0.05-0.14$ ), and tried to quit with NRT but failed (SS; 95\% CI: 0.09; 0.04-0.13).

\section{DISCUSSION}

To our knowledge, this is the first study to investigate which patient factors influence PCP recommendations for e-cigarettes. Using data from a statewide sample, we found that patients' experience with approved cessation medications as well as age and comorbidity were more influential factors than cigarette smoking intensity, intention to quit, interest in approved cessation medications, and experience with e-cigarettes. We found that even during the EVALI outbreak, some physicians held positive views toward e-cigarettes as a smoking cessation aid. Most PCPs in Florida endorsed the use of e-cigarettes for smoking cessation and harm reduction for some of their patients who smoke, particularly among middle-aged patients with comorbidities and those with failed quit attempts that involved using recommended medications. Almost half of the sample reported that they always or often ask patients about e-cigarette use, and nearly one-third at least occasionally recommended ecigarettes to patients who smoke. Despite the context of the EVALI outbreak, these results are consistent with findings from a national survey that found US physicians frequently discuss e-cigarettes in a clinical context, and a substantial proportion have recommended them to their patients. ${ }^{19}$

Although overall support for e-cigarettes among PCPs was not high, our findings suggest that PCPs are more likely to be supportive of e-cigarettes among subgroups of patients.

Table 3 Physician Practices Regarding Tobacco Cessation and E-cigarettes $(N=216)$

\begin{tabular}{|c|c|c|c|c|c|}
\hline \multirow[t]{2}{*}{ Practices } & \multicolumn{5}{|l|}{ Response } \\
\hline & Never & Rarely & Sometimes & Often & Always \\
\hline \multicolumn{6}{|l|}{ Clinical practice guideline adherence } \\
\hline Ask if patient smokes & - & - & $2(0.9 \%)$ & $43(19.9 \%)$ & $169(78.2 \%)$ \\
\hline Advise patients to quit & - & $1(0.5 \%)$ & $3(1.4 \%)$ & $38(17.6 \%)$ & $171(79.2 \%)$ \\
\hline Assess if patient is ready to quit & $1(0.5 \%)$ & $7(3.2 \%)$ & $10(4.6 \%)$ & $68(31.5 \%)$ & $127(58.8 \%)$ \\
\hline Assist patients in quitting & $1(0.5 \%)$ & $2(0.9 \%)$ & $19(8.8 \%)$ & $67(31.0 \%)$ & $124(57.4 \%)$ \\
\hline Arrange follow-up & $8(3.7 \%)$ & $15(6.9 \%)$ & $41(19.0 \%)$ & $61(28.2 \%)$ & $89(41.2 \%)$ \\
\hline Refer to cessation treatment & $30(13.9 \%)$ & $42(19.4 \%)$ & $48(22.2 \%)$ & $38(17.6 \%)$ & $55(25.5 \%)$ \\
\hline \multicolumn{6}{|l|}{ Smoking cessation treatment recommendations } \\
\hline Bupropion (Zyban) & $11(5.1 \%)$ & $29(13.4 \%)$ & $85(39.4 \%)$ & $75(34.7 \%)$ & $10(4.6 \%)$ \\
\hline Varenicline (Chantix) & $5(2.3 \%)$ & $21(9.7 \%)$ & $54(25.0 \%)$ & $106(49.1 \%)$ & $25(11.6 \%)$ \\
\hline Nicotine replacement therapy & $3(1.4 \%)$ & $14(6.5 \%)$ & $61(28.2 \%)$ & $104(48.1 \%)$ & $25(11.6 \%)$ \\
\hline Combination therapy & $17(7.9 \%)$ & $35(16.2 \%)$ & $60(27.8 \%)$ & $81(37.5 \%)$ & $14(6.5 \%)$ \\
\hline Referral to cessation treatment & $38(17.6 \%)$ & $61(28.2 \%)$ & $53(24.5 \%)$ & $33(15.3 \%)$ & $23(10.6 \%)$ \\
\hline E-cigarettes & $102(47.2 \%)$ & $44(20.4 \%)$ & $43(19.9 \%)$ & $20(9.3 \%)$ & $1(0.5 \%)$ \\
\hline Ask patients about e-cigarette use & $16(7.4 \%)$ & $33(15.3 \%)$ & $55(25.5 \%)$ & $50(23.1 \%)$ & $56(25.9 \%)$ \\
\hline Patients ask about e-cigarettes & $19(8.8 \%)$ & $82(38.0 \%)$ & $91(42.1 \%)$ & $15(6.9 \%)$ & $3(1.4 \%)$ \\
\hline Encourage patients to try other cessation methods before e-cigarettes & $18(8.3 \%)$ & $18(8.3 \%)$ & $32(14.8 \%)$ & $65(30.1 \%)$ & $75(34.7 \%)$ \\
\hline
\end{tabular}




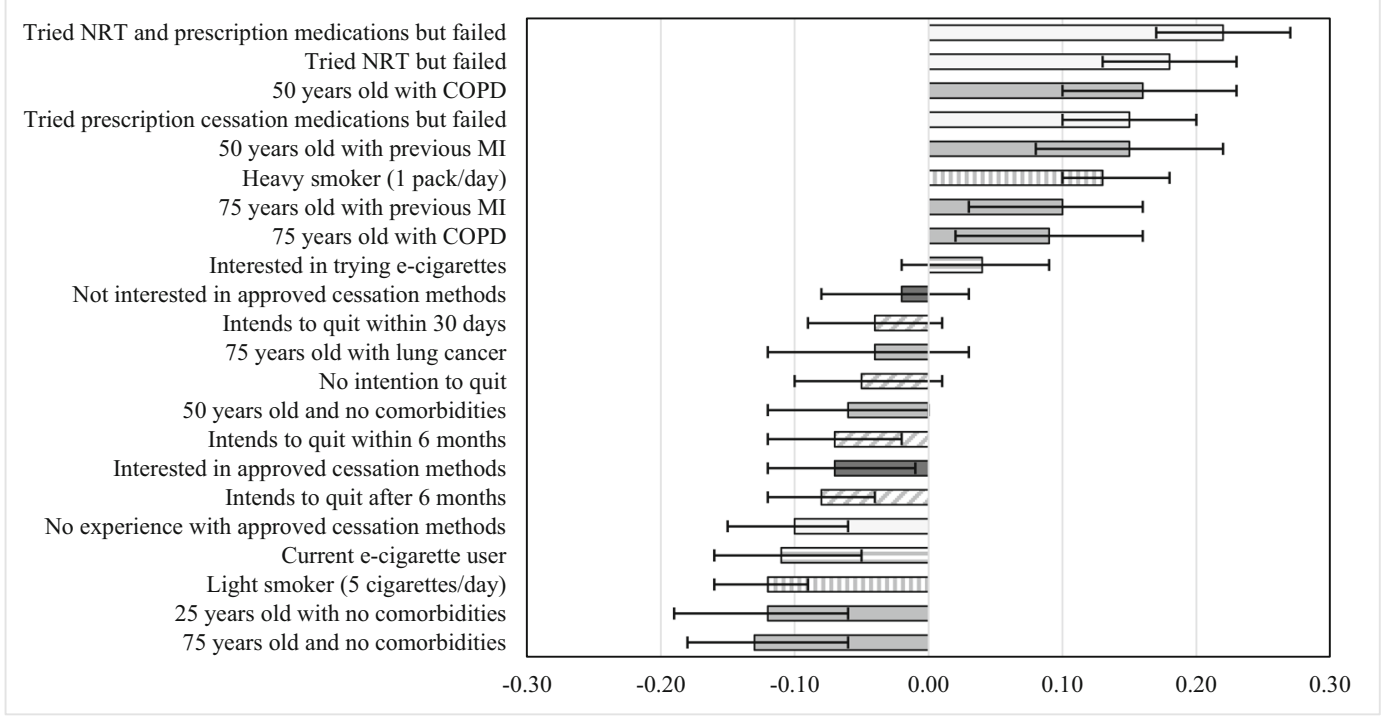

Attributes (all attribute levels are indicated with the same pattern):

$$
\begin{aligned}
& \text { Age and comorbidity } \\
& \text { Smoking intensity }
\end{aligned}
$$

\begin{tabular}{|c|c|}
\hline Experience with approved cessation medications & $\equiv$ Experience with e-cigarettes \\
\hline Age and comorbidity & Interest in approved cessation medications \\
\hline Smoking intensity & Intention to quit \\
\hline
\end{tabular}$$
\text { Experience with approved cessation medications }
$$

Figure 2 Most and least important patient attributes for physician recommendation of e-cigarettes: standardized scores and $95 \%$ confidence intervals. Note: Because the number of attribute levels varied across attributes, standardized scores were calculated for each attribute level by dividing the raw scores (i.e., the difference between the most and least counts, +1 if selected as most important, -1 if least important, and 0 otherwise) by the maximum possible score. Positive scores reflect attributes endorsed as most important to e-cigarettes recommendation, and negative scores reflect attributes endorsed as least important to e-cigarettes recommendation. The bar lengths represent standardized scores and the lines through the bars represent confidence intervals. Attributes (all attribute levels are indicated with the same pattern): experience with approved cessation medications; age and comorbidity; || experience with e-cigarettes; $\bar{\equiv}$ smoking intensity; interest in approved cessation medications; intention to quit

Patients' unsuccessful experiences with approved cessation medications and patient comorbidities were among the most influential factors in the PCPs' recommendations, regardless of whether they recommended e-cigarettes or not. Physicians who recommend e-cigarettes may believe that smokers with a failed quit attempt using traditional cessation medications may be good candidates to try e-cigarettes, whereas physicians who do not recommend them may believe that it is especially important for smokers who had a failed quit attempt to continue using traditional cessation methods. These results are consistent with a previous qualitative study that found PCPs were less concerned about the potential harms of e-cigarettes for highly addicted patients and those with comorbidities. ${ }^{34}$ Some PCPs said they would not recommend them regardless of the patient profile, indicating they may have a blanket policy against ecigarette recommendation. This conclusion is further supported by the fact that among those PCPs who did not recommend ecigarettes for at least one patient profile in the DCE, 56.3\% indicated they never recommend e-cigarettes to their patients.

Only $15 \%$ of PCPs in the sample felt very confident in their ability to counsel their patients on e-cigarettes. Moreover, PCPs reported having limited access to institutional guidance on ecigarettes, and almost $20 \%$ turn to patients for information on e-cigarettes. This is consistent with prior research showing physicians express concerns about being able to stay up to date with the emerging science of e-cigarettes, and many physicians lack the confidence to discuss uncertainties around unknown, longerterm health outcomes associated with e-cigarette use. ${ }^{19,35}$ In a 2013 survey of North Carolina physicians, those who were older, held positive beliefs about e-cigarettes, and documented tobacco treatment counseling were more likely to report recommending e-cigarettes to patients. ${ }^{30}$ Another survey of a national sample of physicians found that males were more likely to recommend ecigarettes and endorse a harm reduction approach. ${ }^{32}$

A majority of PCPs in the sample had unfavorable attitudes toward e-cigarettes. Less than half agreed that e-cigarettes reduce secondhand smoke exposure and nearly half never recommend them. These findings may be partially explained by the EVALI outbreak, ${ }^{48}$ which coincided with our survey data collection. However, over half agreed it was important to offer alternatives to traditional cessation treatments. Overall, despite unfavorable attitudes and concerns about safety, PCPs seem to perceive e-cigarettes as a risk reduction strategy. Therefore, the behavior of PCPs appears to be rationally motivated given the limited evidence they have on the harms and benefits of e-cigarette use among smokers. This highlights 
the importance of shared decision-making and the need to help patients make informed decisions in the face of uncertainty. However, a key factor that influences whether discussions about e-cigarettes occur is whether participants are screened for e-cigarette use. We found that approximately half of PCPs ask often or always about e-cigarette use compared with $78 \%$ always asking about cigarettes. Therefore, approximately half of PCPs do not create the opportunity to educate users on ecigarettes' potential risks, benefits, and alternative options. This underscores the importance of screening for e-cigarettes in light of the increased prevalence of product use and in the event health concerns such as EVALI arise to allow for more timely identification of at-risk patients.

Despite e-cigarettes not generally being recommended in the USA due to uncertainties around their effectiveness for cessation and their long-term health consequences, ${ }^{49,50}$ as well as the general negative attitudes toward them among Floridabased PCPs, we found support among these same physicians for e-cigarettes as a smoking cessation strategy. The National Academy of Science, Engineering and Medicine has found conclusive evidence that e-cigarettes are less harmful than smoking $;{ }^{51}$ furthermore, the FDA has adopted a harm reduction approach to tobacco regulation, ${ }^{52}$ and e-cigarette companies are seeking approval for their products as cessation aids. ${ }^{53}$ Given these dynamics, the potential role of e-cigarettes in the treatment of smokers should be considered further.

This study used a BWS experiment to provide novel information on PCPs' views on e-cigarettes. Strengths include a large, statewide sample of PCPs and application of the DCE approach. Similar to other approaches to stakeholder engagement, the primary limitation of BWS is its focus on stated preferences, or PCPs' perceptions of what patient factors are most and least likely to influence their recommendations. PCP responses may not be reflective of actual practices, and there may be other important factors influencing decision-making that were not measured in this study. An additional limitation was the lessthan-optimal response rate, which may have been partially due to the length of the questionnaire and the relatively low incentive, raising the possibility of nonresponse bias. Additionally, care should be taken when generalizing findings. Our sample is less diverse than the PCP population in Florida and nationwide..$^{54,55}$ Additionally, we focused on PCPs, which could limit the generalizability of our findings to other physician groups. However, PCPs are likely to be more involved in primary prevention cessation discussions with their patients who smoke, and given resource limitations in our study, we opted for greater representation of PCP specialties. Finally, our survey was administered during the EVALI outbreak, which likely influenced responses. However, we did not detect any relevant differences in responses received before and after the outbreak, although this may have been due to sample size limitations. Since then, it has been found that the source of the outbreak was likely due to contaminated THC liquids used in vaping devices, and recommendations for ecigarettes may be more frequent now than at the time of data collection.

\section{CONCLUSION}

These findings, along with findings of increased patient interest and recent trial evidence, highlight the need for guidelines to help PCPs incorporate e-cigarettes into current smoking cessation practices. Our findings highlight the need for interventions to support PCPs in delivering smoking cessation advice to their patients who smoke and to address the uncertainty surrounding the risks and benefits of using e-cigarettes as a cessation aid.

Corresponding Author: Ramzi G. Salloum, PhD; Department of Health Outcomes and Biomedical Informatics, College of Medicine, University of Florida 2004 Mowry Road, Gainesville, FL, USA (e-mail: rsalloum@ufl.edu).

Funding This work was supported by the University of Florida College of Medicine and the University of South Carolina Arnold School of Public Health.

\section{Declarations:}

Conflict of interest: The authors declare that they do not have a conflict of interest.

\section{REFERENCES}

1. Creamer MR, Wang TW, Babb S, Cullen KA, Day H, Willis G, Jamal A, Neff L. Tobacco Product Use and Cessation Indicators Among Adults United States, 2018. MMWR Morb Mortal Wkly Rep. 2019;68(45):10131019.

2. Caraballo RS, Shafer PR, Patel D, Davis KC, McAfee TA. Quit Methods Used by US Adult Cigarette Smokers, 2014-2016. Prev Chronic Dis. 2017;14:E32.

3. Adkison SE, O'Connor RJ, Bansal-Travers M, Hyland A, Borland R, Yong HH, Cummings KM, McNeill A, Thrasher JF, Hammond D, et al. Electronic nicotine delivery systems: international tobacco control fourcountry survey. Am J Prev Med. 2013;44(3):207-215.

4. Etter JF, Bullen C. Electronic cigarette: users profile, utilization, satisfaction and perceived efficacy. Addiction. 2011;106(11):2017-2028.

5. Goniewicz ML, Lingas EO, Hajek P. Patterns of electronic cigarette use and user beliefs about their safety and benefits: an internet survey. Drug Alcohol Rev. 2013;32(2):133-140.

6. Patel D, Davis KC, Cox S, Bradfield B, King BA, Shafer P, Caraballo R, Bunnell R. Reasons for current E-cigarette use among U.S. adults. Prev Med. 2016;93:14-20.

7. Barua RS, Rigotti NA, Benowitz NL, Cummings KM, Jazayeri MA Morris PB, Ratchford EV, Sarna L, Stecker EC, Wiggins BS. 2018 ACC Expert Consensus Decision Pathway on Tobacco Cessation Treatment: A Report of the American College of Cardiology Task Force on Clinical Expert Consensus Documents. J Am Coll Cardiol. 2018;72(25):3332-3365.

8. National Academies of Sciences, Engineering, and Medicine. Public Health Consequences of E-Cigarettes. Washington, DC: The National Academies Press. 2018. https://doi.org/10.17226/24952.

9. Prochaska JJ, Benowitz NL. Current advances in research in treatment and recovery: Nicotine addiction. Sci Adv. 2019;5(10):eaay9763.

10. Hartmann-Boyce J, McRobbie H, Bullen C, Begh R, Stead LF, Hajek P. Electronic cigarettes for smoking cessation. Cochrane Database Syst Rev. 2016;9:CD010216.

11. Kalkhoran S, Glantz SA. E-cigarettes and smoking cessation in realworld and clinical settings: a systematic review and meta-analysis. Lancet Respir Med. 2016;4(2):116-128.

12. Rahman MA, Hann N, Wilson A, Mnatzaganian G, Worrall-Carter L. Ecigarettes and smoking cessation: evidence from a systematic review and meta-analysis. PLoS One. 2015;10(3):e0122544.

13. Hartmann-Boyce $\mathbf{J}$, McRobbie $\mathbf{H}$, Lindson $\mathbf{N}$, Bullen $\mathbf{C}$, Begh $\mathbf{R}$, Theodoulou A, Notley C, Rigotti NA, Turner T, Butler AR, et al 
Electronic cigarettes for smoking cessation. Cochrane Database Syst Rev. 2020; 10:CD010216.

14. Hajek P, Phillips-Waller A, Przulj D, Pesola F, Myers Smith K, Bisal N Li J, Parrott S, Sasieni P, Dawkins L, et al. A Randomized Trial of ECigarettes versus Nicotine-Replacement Therapy. $N$ Engl $\mathrm{J}$ Med. 2019;380(7):629-637.

15. Substance Abuse and Mental Health Services Administration; Office of the Surgeon General. Smoking cessation: a report of the Surgeon General. In. Washington, DC: US Department of Health and Human Services; 2020.

16. McNeill A, Brose LS, Calder R, Bauld L, Robson D. Vaping in England: an evidence update February 2019. London, England: Public Health England;2019.

17. Royal College of Physicians of London (RCP). Nicotine without smoke Tobacco harm reduction. London: RCP;2016. 1860166008.

18. Doescher MP, Wu M, Rainwater E, Khan AS, Rhoades DA. Patient Perspectives on Discussions of Electronic Cigarettes in Primary Care. $J$ Am Board Fam Med. 2018;31(1):73-82.

19. Nickels AS, Warner DO, Jenkins SM, Tilburt J, Hays JT. Beliefs, Practices, and Self-efficacy of US Physicians Regarding Smoking Cessation and Electronic Cigarettes: A National Survey. Nicotine Tob Res. 2017;19(2):197-207.

20. Berg CJ, Haardoerfer R, Escoffery C, Zheng P, Kegler M. Cigarette users' interest in using or switching to electronic nicotine delivery systems for smokeless tobacco for harm reduction, cessation, or novelty: a crosssectional survey of US adults. Nicotine Tob Res. 2015;17(2):245-255.

21. Kollath-Cattano C, Thrasher JF, Osman A, Andrews JO, Strayer SM Physician Advice for e-Cigarette Use. J Am Board Fam Med. 2016;29(6):741-747.

22. Gravely S, Thrasher JF, Cummings KM, Ouimet J, McNeill A, Meng G, Lindblom EN, Loewen R, O'Connor RJ, Thompson ME, et al. Discussions between health professionals and smokers about nicotine vaping products: results from the 2016 ITC Four Country Smoking and Vaping Survey. Addiction. 2019;114 Suppl 1:71-85.

23. Drouin O, McMillen RC, Klein JD, Winickoff JP. E-Cigarette Advice to Patients From Physicians and Dentists in the United States. Am J Health Promot. 2018;32(5):1228-1233.

24. Hung DY, Leidig R, Shelley DR. What's in a setting?: Influence of organizational culture on provider adherence to clinical guidelines for treating tobacco use. Health Care Manage Rev. 2014;39(2):154-163.

25. Jamal A, Dube SR, Malarcher AM, Shaw L, Engstrom MC, Centers for Disease C, Prevention. Tobacco use screening and counseling during physician office visits among adults-National Ambulatory Medical Care Survey and National Health Interview Survey, United States, 2005-2009. MMWR Suppl. 2012;61(2):38-45.

26. Alcala HE, Shimoga SV. It Is About Trust: Trust in Sources of Tobacco Health Information, Perceptions of Harm, and Use of E-Cigarettes. Nicotine Tob Res. 2020;22(5):822-826

27. Siu AL, Force USPST. Behavioral and Pharmacotherapy Interventions for Tobacco Smoking Cessation in Adults, Including Pregnant Women: U.S. Preventive Services Task Force Recommendation Statement. Ann Intern Med. 2015;163(8):622-634.

28. Pepper JK, McRee AL, Gilkey MB. Healthcare providers' beliefs and attitudes about electronic cigarettes and preventive counseling for adolescent patients. J Adolesc Health. 2014;54(6):678-683.

29. England LJ, Anderson BL, Tong VT, Mahoney J, Coleman-Cowger VH, Melstrom P, Schulkin J. Screening practices and attitudes of obstetricians-gynecologists toward new and emerging tobacco products. Am J Obstet Gynecol. 2014;211(6):695 e691-697.

30. Kandra KL, Ranney LM, Lee JG, Goldstein AO. Physicians' attitudes and use of e-cigarettes as cessation devices, North Carolina, 2013. PLOS One. 2014;9(7):e103462.

31. Ofei-Dodoo S, Kellerman $\mathbf{R}$, Nilsen $\mathbf{K}$, Nutting $\mathbf{R}$, Lewis D. Family Physicians' Perceptions of Electronic Cigarettes in Tobacco Use Counseling. J Am Board Fam Med. 2017;30(4):448-459.

32. Steinberg MB, Giovenco DP, Delnevo CD. Patient-physician communication regarding electronic cigarettes. Prev Med Rep. 2015;2:96-98.

33. Gallegos-Carrillo K, Barrientos-Gutierrez I, Arillo-Santillan E, ZavalaArciniega L, Cho YJ, Thrasher JF. Health Professionals' Counseling about Electronic Cigarettes for Smokers and Vapers in a Country That Bans the Sales and Marketing of Electronic Cigarettes. Int $J$ Environ Res Public Health. 2020;17(2):442.

34. El-Shahawy O, Brown R, Elston Lafata J. Primary Care Physicians' Beliefs and Practices Regarding E-Cigarette Use by Patients Who Smoke: A Qualitative Assessment. Int J Environ Res Public Health. 2016; 13(5):445.

35. Kollath-Cattano C, Dorman T, Albano AW, Jr., Jindal M, Strayer SM Thrasher JF. E-cigarettes and the clinical encounter: Physician perspectives on e-cigarette safety, effectiveness, and patient educational needs. J Eval Clin Pract. 2019;25(5):761-768.

36. Damschroder LJ, Aron DC, Keith RE, Kirsh SR, Alexander JA, Lowery JC. Fostering implementation of health services research findings into practice: a consolidated framework for advancing implementation science. Implement Sci. 2009;4:50.

37. Salloum RG, Shenkman EA, Louviere JJ, Chambers DA. Application of discrete choice experiments to enhance stakeholder engagement as a strategy for advancing implementation: a systematic review. Implement Sci. 2017;12(1):140.

38. Buckell J, Sindelar JL. The impact of flavors, health risks, secondhand smoke and prices on young adults' cigarette and e-cigarette choices: a discrete choice experiment. Addiction. 2019;114(8):1427-1435.

39. Czoli CD, Goniewicz M, Islam T, Kotnowski K, Hammond D. Consumer preferences for electronic cigarettes: results from a discrete choice experiment. Tob Control. 2016;25(e1):e30-36

40. Shang C, Weaver SR, White JS, Huang J, Nonnemaker J, Cheng KW, Chaloupka FJ. E-cigarette Product Preferences among Adult Smokers: A Discrete Choice Experiment. Tob Regul Sci. 2020;6(1):66-80.

41. Quaife M, Terris-Prestholt F, Di Tanna GL, Vickerman P. How well do discrete choice experiments predict health choices? A systematic review and meta-analysis of external validity. Eur $J$ Health Econ. 2018;19(8): 1053-1066.

42. Lancsar E, Louviere J, Donaldson C, Currie G, Burgess L. Best worst discrete choice experiments in health: methods and an application. Soc Sci Med. 2013;76(1):74-82

43. McLeod CC, Klabunde CN, Willis GB, Stark D. Health care provider surveys in the United States, 2000-2010: a review. Eval Health Prof. 2013;36(1): 106-126

44. Dillman DA, Smyth JD, Christian LM. Internet, phone, mail, and mixed-mode surveys: the tailored design method. Hoboken, NJ: John Wiley \& Sons; 2014.

45. Muhlbacher AC, Kaczynski A, Zweifel P, Johnson FR. Experimental measurement of preferences in health and healthcare using best-worst scaling: an overview. Health Econ Rev. 2016;6(1):2.

46. The American Association for Public Opinion Research. Standard Definitions: Final Dispositions of Case Codes and Outcome Rates for Surveys, 9th edition. AAPOR. 2016. https://www.aapor.org/AAPOR Main/media/publications/Standard-Definitions20169theditionfinal.pdf. Accessed 15 July 2020.

47. Smith TW. A revised review of methods to estimate the status of cases with unknown eligibility. Chicago: University of Chicago, National Opinion Research Center. 2009

48. Salzman GA, Alqawasma M, Asad H. Vaping Associated Lung Injury (EVALI): An Explosive United States Epidemic. Mo Med. 2019;116(6):492-496.

49. U.S. Preventive Services Task Force. Tobacco Smoking Cessation in Adults, Including Pregnant Persons: Interventions. https://www. uspreventiveservicestaskforce.org/uspstf/draft-recommendation/tobacco-smoking-cessation-in-adults-including-pregnant-womeninterventions\#fullrecommendationstart. Published 2020. Updated June 2, 2020. Accessed July 8, 2020.

50. American Cancer Society. American Cancer Society Position Statement on Electronic Cigarettes. https://www.cancer.org/healthy/stay-awayfrom-tobacco/e-cigarette-position-statement.html. Published 2020. Accessed July 8, 2020

51. National Academies of Sciences, Engineering, and Medicine. Public health consequences of e-cigarettes. Washington, DC: The National Academies Press; 2018.

52. Gottlieb S, Zeller M. A Nicotine-Focused Framework for Public Health. New England Journal of Medicine. 2017;377(12):1111-1114.

53. Juul Labs Inc. Comment. Smoking Cessation and Related Indications. Developing Nicotine Replacement Therapy Drug Products. Draft Guidance for Industry. Dkt No FDA-2019-D-0297. https://www.juullabs. com/wp-content/uploads/2020/06/Juul-Labs-Inc-Comment_SmokingCessation-and-Related-Indications_Developing-Nicotine-ReplacementTherapy-Drug-Products_Draft-Guidance-for-Industry-Dkt-No-FDA2019-D-0297.pdf Published 2019. Accessed June 25, 2020.

54. Florida Department of Health. 2019 Physician Workforce Annual Report. 2019. https://www.floridahealth.gov/provider-and-partner-resources/communityhealth-workers/physician-workforce-development-andrecruitment/ 2019DOHPhysicianWorkforceReport.2.pdf. Accessed 15 July 2020.

55. Petterson S, McNellis R, Klink K, Meyers D, Bazemore A. The State of Primary Care in the United States: A Chartbook of Facts and Statistics. Washington, DC: Robert Graham Center. 2018.

Publisher's Note: Springer Nature remains neutral with regard to jurisdictional claims in published maps and institutional affiliations. 